\title{
Achievement of Functional Independence in a Patient with Sickle Cell Disease with Autoimmune Hepatitis, Osteomyelitis, Wilson's Disease, and Pathological Fracture Following Physiotherapy
}

\author{
Laukik Vaidya ${ }^{1}$, Waqar Naqvi², Abhiram Awasthi ${ }^{3}$, Kiran Kumar', Pratik Phansopkar ${ }^{5}$ \\ 1,2,4,5 Department of Musculoskeletal Physiotherapy, Ravi Nair Physiotherapy College, Datta Meghe Institute of \\ Medical Sciences, Wardha, Maharashtra, India. ${ }^{3}$ Department of Orthopaedics, Jawaharlal Nehru Medical College, Datta \\ Meghe Institute of Medical Sciences, Wardha, Maharashtra, India.
}

\section{INTRODUCTION}

Sickle Cell Anaemia (SCA) is an autosomal recessive condition caused by malfunction in the synthesis of haemoglobin $(\mathrm{Hb})$ where a single amino acid glutamic acid in the $\beta$ globin gene is exchanged with valine. ${ }^{1}$ The red blood cells assume an abnormal biconcave disk into elongated, rigid, crescent-shaped sickle cells, causing haemolysis and occlusion of microcirculation, which then in turn will lead to hypoxia and sickling of the tissue, ${ }^{2}$ reducing their capacity to microcirculation, with frequent clotting and thrombosis, leading to obstruction, ischemia and infarction, damaging many organs. ${ }^{1}$

Auto-Immune-Hepatitis (AIH) has high risk of steroid-related harmful reactions or other immunosuppressive therapies. ${ }^{3}$ Blood transfusions is useful in prevention and treatment of acute and chronic conditions. ${ }^{4}$ The subsequent elevated excretion of bilirubin predisposes to cholelithiasis, which might be attributed to several other hepatological complications such as haemosiderosis related to transfusion, persistent viral hepatitis, and autoimmune liver disease (AILD). ${ }^{5}$

Tissue hypoxia causes a supplementary inflammatory response resulting in higher intramedullary pressure and bone pain leading to osteopenia, fractures of stress, vertebral collapse, and growth anomalies. The combination of ischemic and compromised immunity will trigger osteonecrosis and haematogenous osteomyelitis. ${ }^{2}$ Immunodeficiency leading to splenic dysfunction, tissue infarction and excessive iron content contributes to elevated incidence of vaso-occlusive-crisis (VOC) and haematogenous-osteomyelitis. Medullary bone infarction and necrosis generate an appropriate environment for development and spread of bacteria. Osteomyelitis is commonly described as infection that involves tibia, femur and humeral diaphysis, along with vertebral infection. Salmonella preceded by Staphylococcus aureus are organism causing sickle cell-related haematogenous osteomyelitis. ${ }^{1}$

Sickle Cell Anaemia (SCA) is an autosomal recessive genetic disease where single amino acid; glutamic acid is exchanged by valine in $\beta$ globin gene. Red blood cells assume an abnormal, rigid, and sickle structure, damaging many organs. The combination of ischemic and compromised immunity will trigger osteonecrosis and osteomyelitis. Immunodeficiency leading to splenic dysfunction, tissue infarction and excessive iron content contributes to elevated incidence of VOC and osteomyelitis.

In this case report we present a 25 years old student who was referred to physiotherapy department for further management of a pathological fracture. She has a history of SCA, Wilson's disease, autoimmune hepatitis and osteomyelitis with frequent requirement for blood transfusion.

In this study a patient having sickle cell anaemia, Wilson's disease, autoimmune hepatitis, osteomyelitis and external fixator fracture for displaced mid-shaft implant was able to resume ADLs (Activities of Daily Living) and independent ambulation.
Corresponding Author:

Dr. Kiran Kumar.

Department of Musculoskeletal

Physiotherapy, Ravi Nair Physiotherapy

College, Datta Meghe Institute of Medical

Science, Wardha, Maharashtra, India.

E-mail: kiran.kumar@dmimsu.edu.in

DOI: $10.14260 / \mathrm{jemds} / 2020 / 719$

How to Cite This Article:

Vaidya L, Naqvi W, Awasthi A, et al. Achievement of functional independence in a patient with sickle cell disease with autoimmune hepatitis, osteomyelitis, Wilson's disease, and pathological fracture following physiotherapy. J Evolution Med Dent Sci 2020;9(43):3271-3275, DOI: 10.14260/jemds/2020/719

Submission 13-07-2020,

Peer Review 15-09-2020,

Acceptance 22-09-2020,

Published 26-10-2020.

Copyright (C) 2020 JEMDS. This is an open access article distributed under Creative Commons Attribution License [Attribution 4.0 International (CC BY 4.0)] 


\section{PRESENTATION OF CASE}

In this case report, we present a 25 years old student who was referred to physiotherapy department for further management of a pathological fracture. While doing bed mobility which was assisted by her mother, a loud cracking sound was heard. She was taken to Acharya Vinobha Bhave Rural Hospital (AVBRH), DMIMS (DU), Wardha, Maharashtra, India, where she was diagnosed with pathological fracture (Figure 1, 2). One-month prior, a sequestrectomy was done and around 1.5 litres of pus was exerted, which lead to breaking of bone in the area leading to a compound midshaft femur fracture on the same site from where the pus was extracted. An external fixator was applied (Figure 3, 4, 5, 6) to stabilize the fracture. She has a history of SCA, Wilson's disease, and autoimmune hepatitis and haematogenous osteomyelitis with frequent requirement for blood transfusion. In the past year she was hospitalized several times for concerns such as fever, abdominal pain, vomiting, swelling over the face, chill fever, dry cough, pain in both lower limbs, pain and swelling over the left thigh, swelling of the lower limbs, breathlessness, etc. for which she was undergoing treatment in same hospital.

\section{Clinical Findings}

She was evaluated in supine lying position with both shoulders at the same level with external fixator in left thigh. On inspection, left leg was abducted and slightly externally rotated, knee extended with pillow support below, leg and ankle where ankle was slightly plantar flexed. Swelling was present on left mid-thigh, knee and operative site. Local temperature was increased on palpation and non-pitting oedema (Figure 7) (Table 1, 2).

\begin{tabular}{|ccc|}
\hline Muscles & Right & Left \\
& Hip & \\
\hline Flexors & Poor & Poor \\
Extensors & Poor & Poor \\
Abductors & Poor & Poor \\
Abductors & Poor & Poor \\
& Knee & \\
\hline Flexors & Poor & Poor \\
Extensors & Poor & Poor \\
Plantar Flexors & Ankle & \\
Doris Flexors & Poor & Poor \\
\hline Invertors & Poor & Poor \\
\hline Evertors & Poor & Poor \\
\hline & Poor & Poor \\
\hline
\end{tabular}

\begin{tabular}{|c|c|c|c|}
\hline Joint & Active & Passive & Limitation \\
\hline \multicolumn{4}{|c|}{ Help } \\
\hline Flexion & NA & $\mathrm{NA}$ & Unable to perform due to pain \\
\hline Extension & NA & NA & Unable to perform due to pain \\
\hline Abduction & NA & NA & Unable to perform due to pain \\
\hline Abduction & NA & NA & Unable to perform due to pain \\
\hline \multicolumn{4}{|r|}{ 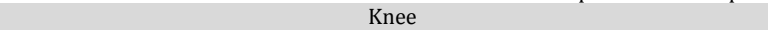 } \\
\hline Flexion & NA & NA & Unable to perform due to pain \\
\hline Extension & NA & NA & Unable to perform due to pain \\
\hline \multicolumn{4}{|r|}{ (6) } \\
\hline Plantar Flexion & $0-20^{\circ}$ & $0-25^{0}$ & NA \\
\hline Doris Flexion & $0-10^{0}$ & $0-15^{0}$ & NA \\
\hline Inversion & $0-15^{0}$ & $0-15^{0}$ & NA \\
\hline Eversion & $0-10^{0}$ & $0-10^{0}$ & NA \\
\hline \multicolumn{4}{|c|}{ Table 2. Range of Motion } \\
\hline
\end{tabular}
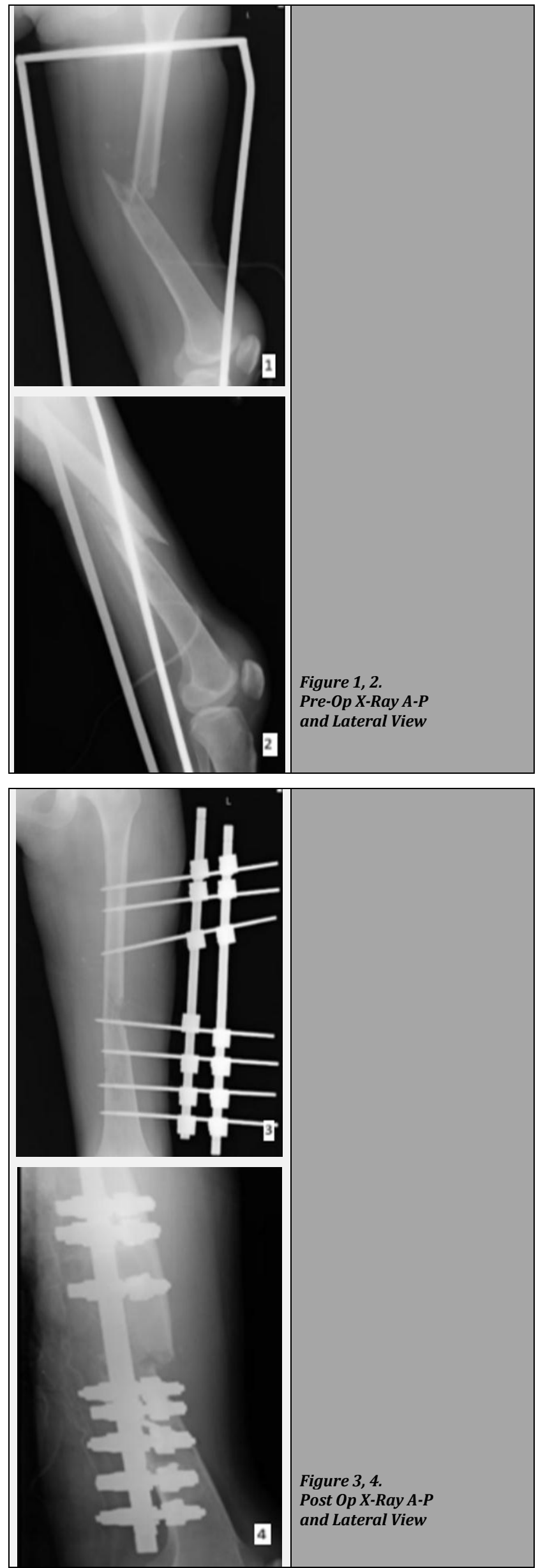


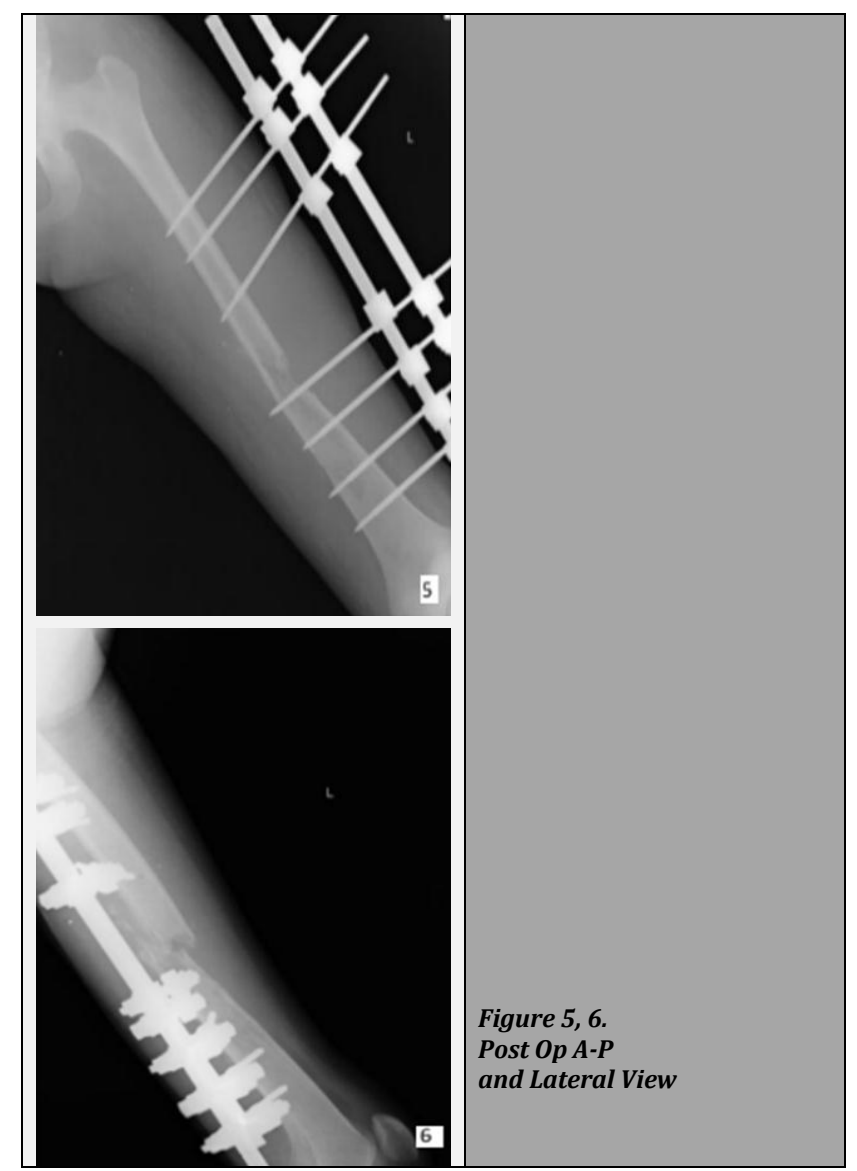

\begin{tabular}{|cc|}
\hline Events & Date \\
Diagnosed with Sickle Cell Anaemia, Wilson's Disease and \\
Autoimmune Hepatitis & $12 / 07 / 2019$ \\
Visited AVBRH with complains of pain swelling over the left thigh & $09 / 04 / 2019$ \\
Diagnosed with Osteomyelitis. & $09 / 03 / 2020$ \\
Sequestrectomy and Saucerization of left tibia and femur. & $11 / 03 / 2020$ \\
$\begin{array}{c}\text { Revisited AVBRH as tired to lift up pelvis when a cracking sound } \\
\text { was heard along with complains of sudden pain inability to move } \\
\text { leg which didn't reduce. }\end{array}$ & $01 / 04 / 2020$ \\
Diagnosed with compound midshaft femur fracture & $01 / 04 / 2020$ \\
External Fixator was applied for compound midshaft femur fracture & $02 / 04 / 2020$ \\
Referred to Physiotherapy for further management & $05 / 04 / 2020$ \\
\hline Figure 7. Timeline \\
\hline \multicolumn{2}{|c}{} \\
\hline
\end{tabular}

\section{DISCUSSION OF MANAGEMENT}

\section{Medical Management}

She is a known patient of sickle cell anaemia, Wilson's disease, autoimmune hepatitis, and haematogenous osteomyelitis with frequent requirement for blood transfusion (haemoglobin: - 4 gm \%), was managed with saline washed PRC transfusions and injectable antibiotics and tablet hydroxyurea once daily, Tablet Folic Acid $5 \mathrm{mg}$ once daily and Tablet Sodamint thrice daily. One-month later patient developed tense red hot swelling around the knee and midshaft femur, for which an emergency incision and drainage of the swellings and 1.5 litres of pus was drained out from the midshaft femur and proximal tibia. Sequestrectomy was done for it. The reports of culture showed growth of staphylococcus aureus with sensitivity to amikacin. The patient was started on injection amikacin for two weeks with regular KFT monitoring and regular blood transfusions.

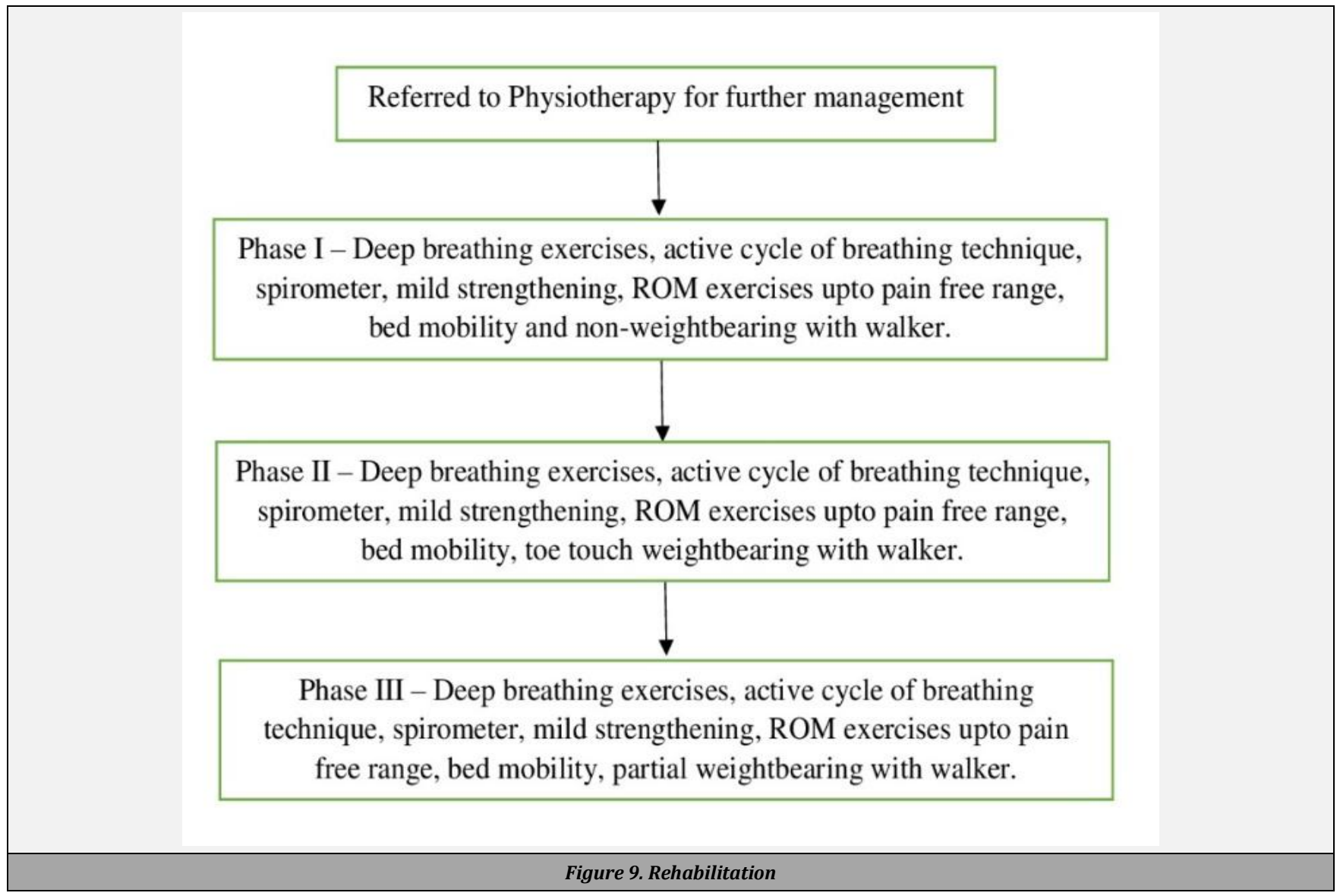


The patient was then discharged on oral ciprofloxacin. After one-month patient again presented with pathological fracture midshaft femur left side. She was managed with realignment of the fracture and external fixator application with three proximal and three distal pins and connected with two connecting rods and injectable antibiotics.

\section{Post-Operative Goals}

The short-term goals were to avoid respiratory problems, minimize pain and oedema, preserve and improve the joint range of motion and strength, facilitate early mobility, prevent pressure sores, facilitate walking (non-weight bearing) and independent ADLs. Long-term goals involved improve joint range of motion and strength, encouraging walking, balance, independent ADL and ergonomics. ${ }^{6-12}$ (Figure 7).

\section{Post-Operative Management}

\section{Phase I (Inpatient. Postoperative: 0 - 4 Weeks)}

Phase I activities concentrate on breathing exercises, joint mobility in the hip and knee, non-weight-bearing strengthening, statics and active movements.

Relaxation techniques, active cycle of breathing technique, deep breathing and spirometry avoid problems in the respiratory system. Movement of the ankle toe to prevent oedema. Static quads, static hams and static glutei which sustain the quadriceps, hamstring, and glutes strength. Half bridging to sustain core strength (using leg and elbows that are not affected). Active exercises for unaffected limbs were continued along with proper positioning and bed mobility to reduce the occurrence of pressure sores, active assisted SLR (Straight Leg Raise) to retain quadriceps and hamstring strength, to decrease joint stiffness and increase range, active assisted hip abduction, adduction to preserve hip abductor and adductor flexibility, to decrease joint stiffness and increase range. Heel slides to retain quadriceps and hamstring strength, decrease joint stiffness and increase range, active quadriceps assisted dynamic. Long sitting and bed side were promoted sitting with or without a bed support. Bed side standing with walker and walking with walker (non-weight bearing) to enhance balance, reduce fear of falling, obtain confidence and strength.

\section{Phase II (Outpatient. Postoperative: 4 - 8 Weeks)}

Most phase I aspects persisted through phase II, as needed. Weight bearing with walker (toe touch). Phase II workouts featured a transformation of phase I exercises with further weight-bearing activities, additional gait retraining, and strength conditioning incorporation.

Resisted exercises were performed with increased motion range, such as SLR, prone SLR, side SLR, heel slides, and dynamic quadriceps. It was encouraged to stand and walk with a walker (partial weight bearing).

\section{Phase III (Outpatient. Postoperative: 8 - 12 Weeks)}

The goal during Phase III is to promote balance, mobility and endurance training. The exercises focused on increasing the strength of the lower extremity as tolerated lower extremity walker activities.
Resisted exercises were done with moderated resistance with increased range of motion such as SLR, prone SLR, side SLR, heel slides, dynamic quadriceps. Standing and walking with walker was facilitated. Gait training exercises such as marching on the spot, stepping, ascending stairs, stride walking retro walking, tandem walking, high step walking to enhance coordination, promoting strength.

\section{Limitations}

As she was confined to bed with less to no bed mobility, the cardiovascular endurance and strength was less. As a result, the patient would get fatigue soon resulting in less output than required which delayed the rehabilitation process of making patient self-dependent.

\section{DISCUSSION}

In this case report, we found, a patient with history of osteomyelitis, for which sequestration was done for left leg with 1.5 litres of pus extracted leading to pathological fracture which was managed with an external fixation. Goals for rehabilitation were set, starting from mild exercises to strengthening and assisted weight bearing ambulation. All the exercises were performed with each one three times a day 10 sets. There are literatures that show the effects of internal fixation and Ilizarov fixation its effect on rehabilitation, but research on external fixation and routine rehabilitation are missing.

Early mobilization and total weight bearing8 in patients with femur fractures was commonly correlated with a quick and stress-free recovery with very little effect on pain, hip mobility and walking capacity. We couldn't mobilize her early as due to weakness due to her prolonged stay in hospital.

As program comprising hip range of motion exercises, isometric exercises and eccentric strengthening as suggested by recent researches, ${ }^{9,10}$ enhances strength of hip flexors, abductors, walking rhythm and cadence. We incorporated isometrics, eccentrics and range of motion exercises.

Functional limitations mostly influence the result as study done by; of patient who underwent femoral fractures included hip abductor weakness, quadriceps femoris muscle weakness and anterior knee pain might relate to an altered gait pattern individually or collectively. ${ }^{11}$ Thus, we enforced more on strengthening them

A flexible protocol was made by our team considering her weakness and to enhance her strength and endurance, 12 sufficient considerations should be offered to exceptional cases with reference to injuries and the rehabilitation protocol, flexibility with certain circumstances might be required; furthermore, all attempt must be made to proceed with a goal-based development of rehabilitation protocol, specific with progress to weight-bearing activities as quickly as possible, since this guides rehabilitation advancement.

\section{CONCLUSIONS}

Rehabilitation program successfully minimizes post-operative pain, oedema, increased strength and range of motion. In this 
study, a patient having sickle cell anaemia, Wilson's disease, autoimmune hepatitis, osteomyelitis, and external fixator fracture, for displaced mid-shaft implant was able to resume ADLs and independent ambulation.

Financial or other competing interests: None.

Disclosure forms provided by the authors are available with the full text of this article at jemds.com.

\section{REFERENCES}

[1] AlDallal SM. Osteomyelitis: a manifestation of sickle cell anemia. Clin Med Investig 2017;2(3):1-3.

[2] Amiri AH, Shekarriz R, Yazdanian M. Analysis of 16 teenage patients with sickle cell anemia and musculoskeletal complications. J NTR Univ Health Sci 2016;5(2):111-4.

[3] Calore E, Marzollo A, Cananzi M, et al. Haploidentical stem cell transplantation cures autoimmune hepatitis and cerebrovascular disease in a patient with sickle cell disease. Bone Marrow Transplant 2018;53(5):644-6.

[4] Braga CCB, Benites BD, de Albuquerque DM, et al. Deferasirox associated with liver failure and death in a sickle cell anemia patient homozygous for the $-1774 \mathrm{delG}$ polymorphism in the Abcc2 gene. Clin Case Rep 2017;5(8):1218-21.

[5] Jitraruch S, Fitzpatrick E, Deheragoda $M$, et al. Autoimmune liver disease in children with sickle cell disease. J Pediatr 2017;189:79-85.e2.
[6] Coglianese DB, Herzenberg JE, Goulet JA. Physical therapy management of patients undergoing limb lengthening by distraction osteogenesis. J Orthop Sports Phys Ther 1993;17(3):124-32.

[7] Krebs NM, Krebs RC, Yaish AM. Femoral osteomyelitis presenting as a pathologic fracture in a 53 year old male: a rare case report. J Orthop Case Rep 2017;7(6):85-8.

[8] Baer $M$, Neuhaus $V$, Pape $H C$, et al. Influence of mobilization and weight bearing on in-hospital outcome in geriatric patients with hip fractures. SICOT J 2019;5:4.

[9] Harikesavan K, Chakravarty RD, Maiya AG, et al. Hip abductor strengthening improves physical function following total knee replacement: one-year follow-up of a randomized pilot study. Open Rheumatol J 2017;11:3042.

[10] Krommes K, Bandholm T, Jakobsen MD, et al. Dynamic hip adduction, abduction and abdominal exercises from the holmich groin-injury prevention program are intense enough to be considered strengthening exercises - a cross-sectional study. Int J Sports Phys Ther 2017;12(3):371-380.

[11] Paterno MV, Archdeacon MT, Ford KR, et al. Early rehabilitation following surgical fixation of a femoral shaft fracture. Phys Ther 2006;86(4):558-72.

[12] Paterno MV, Archdeacon MT. Is there a standard rehabilitation protocol after femoral intramedullary nailing? J Orthop Trauma 2009;23(5 Suppl):S39-46. 